\title{
BMJ Open Study protocol for an online randomised controlled trial among non-treatment seeking problem gamblers: training inhibition in online problem gambling (TRAIN-online) trial
}

To cite: Santiago A, Carré A, Miranda $\mathrm{R}$, et al. Study protocol for an online randomised controlled trial among nontreatment seeking problem gamblers: training inhibition in online problem gambling (TRAIN-online) trial. BMJ Open 2021;11:e051641. doi:10.1136/ bmjopen-2021-051641

- Prepublication history for this paper is available online. To view these files, please visit the journal online (http://dx.doi. org/10.1136/bmjopen-2021051641).

Received 08 April 2021 Accepted 25 October 2021

Check for updates

(C) Author(s) (or their employer(s)) 2021. Re-use permitted under CC BY-NC. No commercial re-use. See rights and permissions. Published by BMJ.

For numbered affiliations see end of article.

Correspondence to

Antoine Santiago;

santiago_antoine@hotmail.fr

\section{ABSTRACT}

Introduction Development of fully internet-based programs could provide a new avenue to improve access to healthcare for problem gamblers. In this project, we aim to assess the efficacy of a web-based cognitive intervention targeting inhibitory control among problem gamblers, using a randomised controlled design. As impaired inhibitory control is involved in self-regulation difficulties in behavioural addictions, it represents a particularly relevant cognitive process to target for an online psychological intervention.

Methods and analysis This will be a single-blinded, randomised, comparative therapeutic web-based, controlled trial. Up to 200 non-treatment seeking adult problem gamblers with a Problem Gambling Severity Index-recent (PGSI-recent) score $\geq 5$ will be included. The intervention will be a computerised cognitive training program targeting inhibitory skills. The comparator, an active control, will be a computerised neutral sensorial program. Both programs will be carried out under similar conditions: biweekly online training for 6 weeks and optional telephone support will be offered to patients for debriefing. The main objective of the study is to assess the clinical efficacy of the online cognitive training program at 6 weeks, measured with the PGSI-recent. The secondary objectives are to assess the efficacy on the gambling behaviour assessed by the account-based gambling data, on the self-reported gambling practice, and on the inhibition performance at the neuropsychological level at 6 , 14 and 52 weeks. We will also assess the acceptability of this program and the preferred level of guidance. Data analysis will be in intention-to-treat.

Ethics and dissemination This randomized controlled trial will be executed in compliance with the Helsinki Declaration, and was approved by the local ethics boards (Comite de Protection des Personnes) in October 2017. The findings will be published in peer-reviewed journals.

Trial registration number NCT03673800.

\section{INTRODUCTION}

Gambling disorder and gambling-related harms, defined as the adverse impacts from gambling on the health and well-being of individuals, families, communities and society, ${ }^{1}$ represent a major challenge in

\section{Strengths and limitations of this study}

- This study assesses the clinical efficacy of an innovative web-based intervention of cognitive training in problem gambling.

- Efficacy is documented from different perspectives: clinical ones, that is, subjective patient-reported outcomes and objective account-based gambling data, and neuropsychological assessments.

- An optional guidance by phone performed by a trained neuropsychologist is proposed and focuses on the transferability of the inhibitory control tasks in the patient's real-life situations related to selfregulation difficulties.

- Completion of an online neuropsychological assessment (using a Stop Signal Task) without face-to-face contact is a challenge and limits the interpretation of the participant's cognitive abilities.

public health. Despite guidelines for responsible gambling standards, ${ }^{2}$ the prevalence of gambling disorder is on the rise and was estimated in 2014 at $1.9 \%$ of the general French population aged $15-75 .^{3}$ The most popular gambling games in France are lottery games, far ahead of horse or sports betting, casino and poker. Two million French people gamble online, the majority of whom are young men $(75.8 \%)$, and $45.4 \%$ of online gamblers are under 35 years old versus $31 \%$ of offline gamblers. The development of online gambling could be linked to the increasing role of the internet and new technologies, particularly during the COVID-19 crisis. Indeed, a recent review showed an increase in online gambling during the pandemic for three groups: younger gamblers, male gamblers and gamblers with higher severity of problem gambling. ${ }^{4}$ More generally, online 
gambling may be more likely to contribute to problem gambling than offline environments. ${ }^{5}$

Despite these data, the treatment gap is concerning: according to the Observatoire Des Jeux (French monitoring centre for gambling) national survey, ${ }^{6}$ only $2 \%$ of French problem gamblers seek medical care. Selfstigma and unawareness of professional sources of help have been described as barriers to access the healthcare system in those with gambling disorder. ${ }^{7}$ No medication is currently approved for the treatment of gambling disorder. The most established interventions are motivational ones, cognitive behavioural therapies (CBTs), or a combination of both techniques, but all have demonstrated limited effect size in published trials. ${ }^{8}$ Alternative online interventions among the most at-risk online gamblers could enhance the efficacy of the existing strategies and widen the range of existing sources of help. ${ }^{10}$ A recent study has shown no between-group difference with placebo of fully online CBT among non-treatment seeking problem gamblers. ${ }^{11}$ In this project, we will propose an alternative online intervention of cognitive training among problem gamblers.

Cognitive training is a type of cognitive remediation used to improve neuropsychological functioning due to its supposed malleability and its relation to daily activities. ${ }^{12}$ Contrary to CBT, cognitive training targets specific neurocognitive functions, such as attention, memory or executive functions, rather than cognitive distortions. Cognitive training is currently used in several neuropsychiatric conditions and several studies have supported the possibility of generalisation of trained skills to daily life activities. ${ }^{13-17}$ However, very few cognitive training programs have been published and tested in addictive disorders. ${ }^{18}$ Content of assessed programs was heterogeneous, and usually targeted multiple executive functions: attention, working memory and spatial orientation, ${ }^{19-21}$ visual-motor coordination and visual-spatial skills. ${ }^{22}$ Most of them reported direct neuropsychological outcomes, while some showed parallel evolution in non-strictly cognitive outcomes, that is, well-being and craving. ${ }^{21-23}$

Volkow and Morales ${ }^{24}$ demonstrated the therapeutic potential in addiction, including gambling disorder, of cognitive training that targets and improves selfregulation skills. ${ }^{24}$ The most explored interventions are cognitive bias modification and cue-specific motor response inhibition, ${ }^{25}$ which are considered specific tasks using addiction-related stimuli. However, Noël et $a t^{26}$ showed a significant effect of non-specific inhibition tasks on decision-making in patients with alcohol use disorder and problem gamblers. ${ }^{26}$ Thus, training on tasks unrelated to any substance or addictive behaviour should lead to both improvement of the addiction itself and better transferability of the enhanced skills to other behaviours and contexts as they are not limited by addiction-related stimuli but target general and transdiagnostic psychological processes. ${ }^{27}$ In a recent study, Penolazzi $e t a l^{28}$ tested the transdiagnostic hypothesis of inhibitory control deficits in gambling disorders. ${ }^{28}$ The results show preserved memory inhibition and impaired motor response inhibition, a pattern of deficits opposite to that previously reported for substance used disorders. These findings suggest that cognitive training targeting motor and visuospatial inhibitory control could be more adapted to online gamblers.

Fully internet-based randomised controlled trial targeting inhibitory control is an emerging design that could be particularly relevant and acceptable in problem gamblers, for whom the internet is the medium of addictive behaviour. ${ }^{29}$ We propose a web-based, randomised, controlled, single-blinded clinical trial, assessing the efficacy of a cognitive training program targeting inhibition, in gamblers older than 18 years old and with a Problem Gambling Severity Index-recent (PGSI) $\geq 5$.

\section{Aims and objectives}

\section{Primary objective}

The main objective of the study is to assess the clinical efficacy of an online computerised cognitive training program targeted on cognitive control, namely on inhibitory control.

\section{Secondary objectives}

The secondary objectives of the study are:

1. To assess the efficacy on the evolution of the gambling behaviour assessed by the account player-based gambling data, at 6, 14 and 52 weeks from baseline. Gambling behaviour includes: total deposit, compulsivity (defined three consecutive deposits within 12 hours), number of deposit in the hour following the stake, total loss per game, number of sessions (a session is defined as a gambling behaviour where the beginning of a session starts when a gambling action occurs after no gambling action for at least $30 \mathrm{~min}$, and the end of the session is a gambling action followed by no gambling action for $30 \mathrm{~min}$ ), session duration and gambling time slot.

2. To assess the efficacy on the evolution of the selfreported gambling practice, and of quality of life at 6 and 14 weeks from baseline.

3. To assess the efficacy on the evolution of inhibition performance at the neuropsychological level at 6 and 14 weeks from baseline.

4. We will also assess the acceptability of this programme and the preferred level of guidance of the nontreatment seeking problem gamblers according to participation in training sessions.

\section{METHODS AND ANALYSIS \\ Study design}

Our study is a therapeutic web-based, comparative, randomised controlled trial, 2 arms, single blinded, with 52 weeks of follow-up. Data will be collected from clinical assessments at baseline, and weeks 6 and 14, and gambling account based data extracted from the French online gambling regulation authority (ANJ) at baseline, weeks 6,14 and 52 . The ANJ is the regulatory authority 


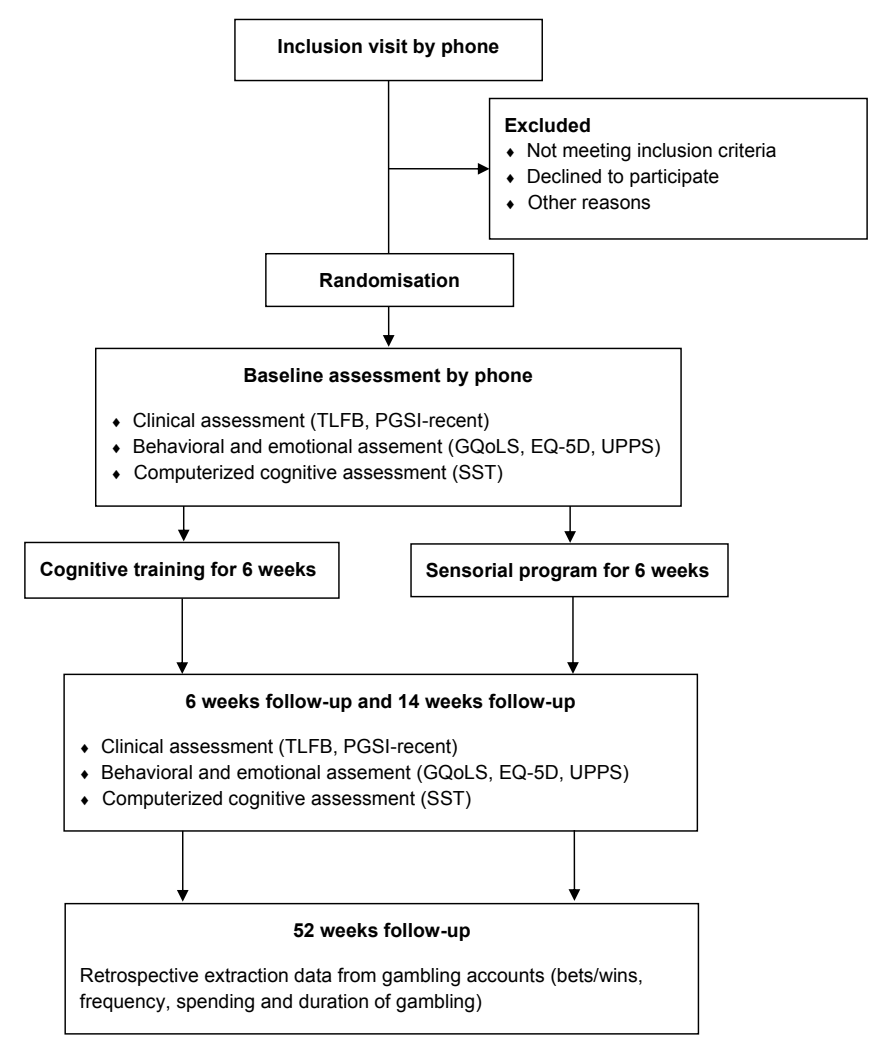

Figure 1 Consolidated Standards of Reporting Trials-flow diagram summarising the trial design with the collected measures and time of collection. EQ-5D, EuroQol five dimensions questionnaire; PGSI, Problem Gambling Index Severity; GQoLS, Gambling Quality of Life Scale; SST, Stop Signal Task; UPPS, multidimensional impulsivity scale.

supervising online gambling in France. It approves and controls all online gambling games and stores the player account data of all online gaming operators. With participant consent, only player account data from legal online gaming operators (approved by the ANJ) will be extracted. Participants who do not have a player account from an approved gaming operator will be included in the study, but no player account data will be extracted for them. The Consolidated Standards of Reporting Trials flow chart of the trial is depicted in figure 1 .

\section{Sample selection}

Both willing gambling operators regulated by the ANJ as well as the ANJ itself will publish a communication on their websites to promote the study. The communication will also be promoted in newspapers, radio programs, gambling online forums and online social media platforms (Facebook, LinkedIn, Instagram). All participants $(\mathrm{n}=200)$ will be enrolled over 20 months using an online procedure. Inclusion criteria will be: (1) over 18 years old gamblers, (2) willing to share his/her first name, last name, exact birthdate and exact place of birth (city +department). This information is needed to extract ANJ player account-based gambling data, to avoid any doubloon or homonym, (3) Canadian Problem Gambling IndexPGSI-recent $\geq 5$ (recall period of 1 month), (4) affiliated to or beneficiary of the French social security system and (5) resident in France. The only non-inclusion criterion will be gamblers who cannot speak or understand French.

\section{Randomisation and group allocation}

A single-blind randomisation will be made by a medical doctor investigator via a central web-based system called Cleanweb. Cleanweb is a secure web-based system used for randomisation and research data storing. Research data, including adverse events, is thus stored in an electronic case report form. Treatment (cognitive training or control intervention) will be allocated according to a computer-generated randomisation list with a 1:1 ratio, using blocks of random size. Only the investigators know which participants are in the cognitive training or control intervention group.

\section{Screening and obtaining consent}

There is no screening visit. Any gambler willing to participate in the study will have to contact the medical doctor investigator by email, who will send back the information notice. In the same email, the investigator will request their telephone number in order to perform the inclusion visit by phone.

Consent will be obtained in a two-step process: an oral consent by phone and an online confirmation in the webbased system Cleanweb. After being given all the relevant study information (study purpose, design, scheduling, intervention, following steps, data collection processes) the person's free and informed oral consent will be obtained by the medical doctor during the inclusion visit by phone. Then, if the inclusion and exclusion criteria are fulfilled, the person will be called back within 3 days by a neuropsychologist investigator to complete the initial assessment (baseline) in Cleanweb. Prior to completing the questionnaires in Cleanweb, the participant will confirm their consent by ticking a box indicating that they freely accept to participate.

\section{Trial flow}

In a first call, a medical doctor will inform the participant about the study, collect consent, check for inclusion and exclusion criteria and the randomisation. Next, a neuropsychologist will call (within 3 days) the participant to present the online data collection process. For data collection, a link will be sent by email to the participant to complete the online questionnaires. The link will only allow the participant to confirm or not their consent, complete the questionnaires and store the data. It will not give access to other information about the study such as the randomisation group. After completing all questionnaires, the participant will be presented with the training program corresponding to randomisation group (blinded). 
Each participant should use the online training twice a week at home via internet, for 6 weeks. The recommended duration for one online training session is $30 \mathrm{~min}$. An optional debriefing by phone is proposed in both groups, up to $15 \mathrm{~min}$ twice a week. The guidance will follow a semi-structured framework including a focus on the emotion associated with the task completion and a focus on the transferability of the tasks in the patient's real life. The neuropsychologist will have access to participants' performances through the therapist interface on the training program.

All follow-up data collection at baseline +6 weeks ( \pm 1 week) and baseline +14 weeks ( \pm 2 weeks) will be made by internet. Automatic email and text-message reminders will be sent to participants to invite them to complete the online assessments at the right time. Non-responders will be contacted by phone to avoid being classified as lost to follow-up.

Account player-based gambling data (last 4 weeks before endpoint, provided by week for each criterion) will be retrospectively extracted from the ANJ database 52 weeks after inclusion.

\section{Interventions}

Experimental intervention

The cognitive training is a computerised cognitive training targeting inhibitory control of motor response, developed in collaboration with a software provider for neuropsychological applications (Scientific Brain Training). It is derived from one of their existing validated programs called 'PRESCO' (Programme de REéducation et de Stimulation COgnitive).$^{30}$ Scientific Brain Training and Paris University Hospital (Assistance Publique-Hôpitaux de Paris, AP-HP) are co-owners of this program. There is then no fee to access it. The tasks included in this program have been selected and modified to target inhibition and are adapted to the population of gamblers whose executive impairments are lower than those encountered in substance use disorders. ${ }^{31}$ More challenging tasks avoid ceiling effect and could thus enhance patients motivation to progress over the training. The tasks are contextualised and gamified. They are non-specific tasks, which do not have gambling-related stimuli. Indeed, the experimental intervention focuses on the training of the general inhibitory control ability, which is supposed to play a role not only in gambling behaviours but also in other self-regulation difficulties related to daily life. Two screen captures from the cognitive program can be seen in figure 2.

A link will be sent by email to the participant to instal the software on their computer. The participant will access the cognitive program with a login identifier created by the neuropsychologist. Participants will be able to access the program at any time, but must train twice a week for an advised duration of $30 \mathrm{~min}$, for 6 weeks. During training sessions, the participant will be able to choose one or more tasks to perform. Debriefing calls will be proposed by the neuropsychologist, according to the participant's

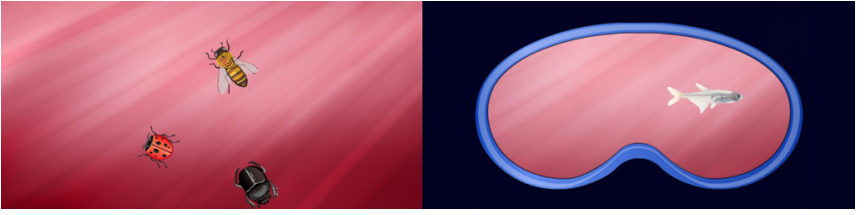

Figure 2 Represents screen captures of two exercises from the active program. The left screen capture is from 'Catch the ladybird'; the right screen capture is from 'Gulf Stream'. Instructions are described in the experimental intervention section.

wishes. Up to two $15 \mathrm{~min}$ scheduled appointments a week will be planned.

The names and the instructions for the six tasks are the following:

- 'Catch the ladybird': 'click as fast as possible on the ladybird that appears at random on your screen. Here, the challenge is that the more ladybirds you catch, the smaller and faster they become! Multiple challenge levels make this even more fun. You will need to focus on the task at hand and resist any distraction that might arise'.

- 'Find your way': 'a trail made up of stones will light up at random and you must memorize the path it creates. This exercise requires you to reproduce the itinerary alternatively from the beginning to the end and from the end to the beginning'.

- 'Under pressure': 'you have to determine the distance between two objects, by quickly scanning the whole screen, and avoiding a color-like distractor'.

- 'Gulf Stream': 'to click as fast as possible on a targetfish previously memorized and avoid clicking on close distractor-fishes crossing the screen'.

- 'Don't fall in the trap': 'to click on target-backboards avoiding close distractor-backboards'.

- 'Color and word Stroop task': 'In the first trial, the written color name differs from the color ink it is printed in, and the participant must say the written word. In the second trial, the participant must name the ink color instead'.

Twelve predetermined levels will be available for every exercise: from the simplest to the most difficult.

\section{Control intervention}

The control intervention consists in a sensorial program with a similar format that targets visual acuity. Two screen captures from the sensorial program can be seen in figure 3. This is not a cognitive program per se and can be considered as neutral in the addiction field.

Access to the sensorial program as well as the duration and format of the training follow the same procedures as for the experimental group.

The following six tasks have been selected in the program 'Action Vision':

- 'Recognize a test pattern': 'to locate and to recognize the test pattern and select one of 3 propositions'. 


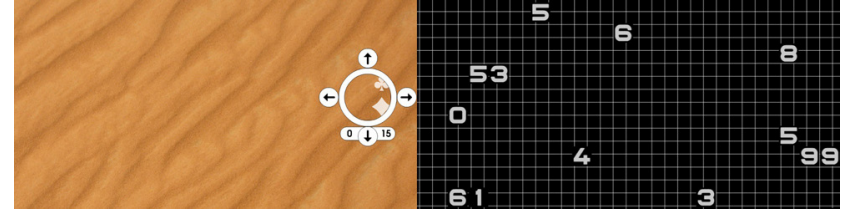

Figure 3 Represents screen captures of two exercises from the control program. The left screen capture is from 'The magnifying glass'; the right screen capture is from 'Click on the target'. Instructions are described in the control intervention section.

- 'Recognize a moving test pattern': 'to locate and to recognize the test pattern before the posting of 3 alternative answers'.

- 'Counting of stationary test patterns': 'to count the test pattern before the posting of 3 alternative answers'.

- 'Counting of moving targets': 'to count moving targets before the posting of 3 alternative answers'.

- 'Click on the target': 'to situate and to click as fast as possibly on the target'.

- 'The magnifying glass': 'to search with the magnifying glass and to click'.

Ten predetermined levels will be available for every exercise: from the simplest to the most difficult.

\section{Measurement instruments}

The primary outcome measure is the change over 6 weeks in the PGSI-recent, a French translation and modified version of the PGSI $^{32}$ with a 30-day recall period, self-completed online in Cleanweb. PGSI has been identified as a tool to measure change in problem gambling. ${ }^{33}$ The original scale has a 12-month recall period. This period was shortened to 30 days for our study. The PGSI consists of nine items which are assessed on a four-point scale: never (1), sometimes (2), most of the time (3) and almost always (4). The total score ranges from 0 to 27 .

Secondary outcomes will be to assess evolution between baseline (T0), 6 weeks (T1) and 14 weeks (T2) of:

- The short form of the multidimensional impulsivity scale named UPPS-P that assesses Urgency, Premeditation, Perseverance, Sensation seeking, and Positive urgency, (UPPS-P Impulsive Behaviour Scale). ${ }^{34} 35$

- TimeLine Follow Back-gambling (money and time including offline gambling).

- EuroQol five dimensions questionnaire. ${ }^{36}$

- Gambling Quality of Life Scale (adapted from Alcohol quality of life scale, ongoing study). ${ }^{37}$

- Neuropsychological assessment: Stop Signal Task measuring cognitive inhibition (stop signal reaction time criteria). ${ }^{38}$

- We will extract from the ANJ database (data collected automatically and prospectively) the following account player based gambling data, at baseline, 6 weeks, 14 weeks and 52 weeks (last 4 weeks): Total Deposit, Total stake per game, Compulsivity (as defined by three consecutive deposits within 12 hours), Number of deposits in the hour following a stake, Total loss per game, Number of sessions (all games): a session is defined as gambling behaviour, whereby the beginning of a session is defined when a gambling action occurs after no gambling action in at least the last $30 \mathrm{~min}$, and the end of the gambling session is a gambling action followed by no gambling action for $30 \mathrm{~min}$ ), Session duration (poker only), Gambling time slot (a: 0:00 to 3:59, b: 4:00 to 7:59, c: 8:00 to $11: 59$, d: $12: 00$ to $15: 59$, e: $16: 00$ to $19: 59$, f: 20:00 to 23:59). The acceptability of this programme will be assessed by the number and length of training sessions and dropout rate.

- Level of guidance will be assessed by the number and length of debriefing calls. We assume that number and length of calls represent intensity criteria and are considered as a change factor.

\section{Estimating expected effect sizes and required sample size}

The sample size was based on the following assumptions on the PGSI: between group change difference: 3 points, estimated SD of the change: 5 points, loss to follow-up at 6 weeks: $55 \%$ maximum. With a power of $80 \%$, a two-sided type I error rate of $5 \%, 200$ patients must be included (100 in each group).

\section{Program dropouts}

Anticipated 55\% maximum for loss to follow-up at 6 weeks. Except for those who withdraw their informed consent, all participants allocated to either study condition will be included in intention-to-treat (ITT) analyses.

\section{Data analysis}

The analysis will include all randomised patients (ITT population). Statistical analyses will be performed with SAS software V.9.2 (SAS Institute, Cary, North Carolina). All primary and secondary analyses will also be performed in the modified ITT population, defined as all randomised patients who attend at least one training session. A multiple imputation approach will be used to replace missing values where appropriate for primary and all secondary outcomes. We will create 10 copies of the dataset, with the missing values replaced by imputed values, based on observed data including outcomes and baseline characteristics of participants. Each dataset will be analysed using standard statistical methods, and the results from each dataset will be pooled into a final result using Rubin's rule.

\section{Analysis of the primary outcome}

The change in PGSI-recent total score over 6 weeks will be compared with the student's t-test. A Wilcoxon test will be applied if data are non-normally distributed.

\section{Analysis of secondary outcomes}

The evolution over time of secondary outcomes will be compared with a linear mixed model. Fixed effects introduced in the model will be time, randomisation group and interaction between time and randomisation group. 
The need for a model with random intercept and slope (vs random intercept only) will be assessed at the time of the analysis with a likelihood ratio test. An appropriate modelling of time will be performed if its effect is not linear. According to Sekhon $e t a l^{39}$, "if an intervention is considered acceptable, patients are more likely to adhere to treatment recommendations and to benefit from improved clinical outcomes' ${ }^{39}$ Thus, we consider the number and the length of training sessions and dropout rate as proxies for acceptability. Indeed, we assume that if the patient perceived the program as effective, he would implant the intervention in his daily life. According to Simons and Kursawe ${ }^{40}$, feasibility is "the proportion of patients who were offered treatment who completed and the number of sessions attended'. ${ }^{40}$ Thus, we will use the number of training sessions and the number of debriefing calls as a measure of feasibility. The number and the length of training sessions, the dropout rate and the number of debriefing calls will be described in each arm, and compared using t-tests or Wilcoxon tests, if data are non-normally distributed.

\section{Patient and public involvement}

Patients were not involved in designing and conducting this research. The French online gambling regulation authority (ANJ) and the willing gaming operators regulated by it are involved in the recruitment process by sharing a communication about the study on their websites. They also share player account data collected during the study (up to 52 weeks after inclusion). Scientific Brain Training provides the experimental and control programmes (which have been adapted for the study) and the software associated.

\section{Monitoring}

The sponsor (AP-HP, Clinical Research and Innovation Department) will monitor the study with a frequency depending on inclusion rates: two or three times a year.

\section{Ethics and dissemination}

This randomised controlled trial will be executed in compliance with the Helsinki Declaration, and was approved by the local ethics board (Comité de Protection des Personnes) in October 2017. All professionals will attend a course in Good Clinical Practice (GCP) and get certified by the Groupement Interrégional de Recherche Clinique et d'Innovation d'île-de-France (public organism providing GCP training). The findings will be published in peer-reviewed journals.

\section{Current trial status}

Recruitment of participants started in February 2019. The last participant is expected to reach the primary endpoint (12-week follow-up) in January 2022. Primary data analysis will begin in March 2022 and the naturalistic follow-up phase of the trial will continue until October 2022 (52 weeks after the last inclusion).

\section{DISCUSSION}

In this article, we describe the protocol of our innovative web-based intervention of cognitive training targeting inhibitory control, with a sensorial program as a comparator to assess its efficacy. The integration of new technologies in therapeutic settings to develop e-health and online interventions represents an interesting alternative to classical psychological interventions. Indeed, although classical interventions such as CBT have been shown to be effective in treating gambling disorder, gamblers make little use of these services. ${ }^{41-44}$ Inhibitory control training is an emerging intervention focusing on a psychological process known to be impaired in different psychiatric conditions. ${ }^{28}$ Thus, as a cognitive endophenotype and vulnerability marker, inhibitory control could be trained with durable effects on behavioural addiction and any associated mental disorder from a transdiagnostic and dual therapeutic perspective. ${ }^{27} 2845$ Moreover, our program could facilitate access to mental services for populations geographically distant from healthcare facilities or living in a context of movement restrictions, as it is currently the case during the COVID-19 pandemic.

Despite these benefits, some risks and limitations must be considered for our online study. Particular care will be taken during the first calls, when included participants will be initiated to their attributed programme application, to the data collection platform, and motivated to complete all assessments including neuropsychological ones. To prevent high dropout rates and non-compliance issues, automatic reminders will help gamblers to complete follow-up assessments, and phone calls will be made to motivate participants in assessment completion if necessary. Guidance will be available according to the participant's wishes, learning from our previous findings suggesting possible adverse effects of imposed guidance among problem gamblers participating in an online clinical trial. ${ }^{11}$ Moreover, completion of neuropsychological assessments without face-to-face contact is a challenge. A cautious analysis of the whole group will be performed to document parameters of the task in this special setting. We will recommend completing the assessments from the same computer, with similar conditions of internet access at the three time points. Another limitation is that we cannot know why some participants refuse the debriefings. We will therefore be cautious about the conclusions drawn from the statistical analyses of guidance. We will also take into consideration the influence of COVID-19 pandemic on gambling behaviour ${ }^{4}$ with secondary analyses of the sociodemographic and gambling characteristics of gamblers included during the lockdowns in France.

If summary, this intervention and its modality without requirement for face-to-face contact could be a tremendous opportunity to help problem gamblers and reduce the treatment gap. 
Author affiliations

${ }^{1}$ Addiction Team, CESP, Villejuif, Île-de-France, France

${ }^{2}$ Department of Addictology, Nîmes University Hospital, Nimes, Languedoc-

Roussillon, France

${ }^{3}$ LIP/PC2S, Université Savoie Mont-Blanc, Chambery, Rhône-Alpes, France

${ }^{4}$ Adult Neurodeveloppment Center, Department of Psychiatry, Pitié-Salpêtrière Hospital, AP-HP, Paris, Île-de-France, France

${ }^{5}$ Department of Psychiatry, Georges Pompidou European Hospital, AP-HP, Paris, Île-de-France, France

${ }^{6}$ U1266, Institute of Psychiatry and Neurosciences of Paris, Paris, Île-de-France, France

${ }^{7}$ Department of Psychiatry, Louis-Mourier Hospital, AP-HP, Colombes, Île-de-France, France

${ }^{8}$ Department of Psychiatry and Addictology, Paul Brousse Hospital, AP-HP, Villejuif, Île-de-France, France

\section{Twitter Arnaud Carré @Arno_Carre}

Acknowledgements The sponsor is Assistance Publique - Hôpitaux de Paris (APHP, Clinical Research and Innovation Department). We would like to thank Sarah Kabani for proofreading the manuscript.

Contributors All authors were involved in conception and trial design. AL, AS and $A C$ wrote the first draft of the paper and revised the manuscript for relevant scientific content. All authors approved the final version of the manuscript. AL provided statistical expertise. All authors are or will be involved in acquisition, analysis and interpretation of data.

Funding This research is support by a call for tenders from IReSP 2015 (Institut de Recherche en Santé Publique) through one of these partners, the ODJ (Observatoire Des Jeux) with a financial contribution from ANJ (French online gambling regulation authority). The grant number for this funder is $\mathrm{N}^{\circ} \mathrm{IreSP} / \mathrm{GC} / \mathrm{SB} / 110$.

Competing interests None declared.

Patient and public involvement Patients and/or the public were not involved in the design, or conduct, or reporting, or dissemination plans of this research.

Patient consent for publication Not applicable.

Provenance and peer review Not commissioned; externally peer reviewed.

Open access This is an open access article distributed in accordance with the Creative Commons Attribution Non Commercial (CC BY-NC 4.0) license, which permits others to distribute, remix, adapt, build upon this work non-commercially, and license their derivative works on different terms, provided the original work is properly cited, appropriate credit is given, any changes made indicated, and the use is non-commercial. See: http://creativecommons.org/licenses/by-nc/4.0/.

\section{ORCID iDs}

Antoine Santiago http://orcid.org/0000-0001-6533-1896

Cédric Lemogne http://orcid.org/0000-0002-3487-4721

\section{REFERENCES}

1 Wardle H, Reith G, Langham E, et al. Gambling and public health: we need policy action to prevent harm. BMJ 2019;365:11807.

2 National council on problem gambling. Internet responsible gambling standards, 2012.

3 Costes J-M, Pousset M, Eroukmanoff V. Les niveaux et pratiques des jeux de hasard et d'argent en 2010. Tendances 2011;77.

4 Hodgins DC, Stevens RMG. The impact of COVID-19 on gambling and gambling disorder: emerging data. Curr Opin Psychiatry 2021;34:332-43.

5 Griffiths M, Wardle H, Orford J, et al. Sociodemographic correlates of internet gambling: findings from the 2007 British gambling prevalence survey. Cyberpsychol Behav 2009;12:199-202.

6 Costes J-M, Eroukmanoff V, Richard J-B. Les jeux d'argent et de hasard en France en 2014. ODJ 2015.

7 Gainsbury S, Hing N, Suhonen N. Professional help-seeking for gambling problems: awareness, barriers and motivators for treatment. J Gambl Stud 2014;30:503-19.

8 Gainsbury S, Blaszczynski A. A systematic review of Internetbased therapy for the treatment of addictions. Clin Psychol Rev 2011;31:490-8.

9 Fink A, Parhami I, Rosenthal RJ, et al. How transparent is behavioral intervention research on pathological gambling and other gambling- related disorders? A systematic literature review. Addiction 2012;107:1915-28

10 Rodda S, Lubman DI. Characteristics of gamblers using a national online counselling service for problem gambling. J Gamb/ Stud 2014;30:277-89.

11 Luquiens A, Tanguy M-L, Lagadec M, et al. The efficacy of three modalities of Internet-based psychotherapy for Non-TreatmentSeeking online problem gamblers: a randomized controlled trial. J Med Internet Res 2016;18:e36.

12 Jaeggi SM, Buschkuehl M, Jonides J, et al. Short- and longterm benefits of cognitive training. Proc Natl Acad Sci U S A 2011;108:10081-6.

13 Klingberg T. Training and plasticity of working memory. Trends Cogn Sci 2010;14:317-24.

14 Katsumi A, Hoshino H, Fujimoto S, et al. Effects of cognitive remediation on cognitive and social functions in individuals with schizophrenia. Neuropsychol Rehabil 2019;29:1475-87.

15 Ashley MJ, Ashley J, Kreber L. Remediation of information processing following traumatic brain injury: a community-based rehabilitation approach. NeuroRehabilitation 2012;31:31-9.

16 Nelson LA, Macdonald M, Stall C, et al. Effects of interactive metronome therapy on cognitive functioning after blast-related brain injury: a randomized controlled pilot trial. Neuropsychology 2013;27:666-79.

17 Bucci P, Piegari G, Mucci A, et al. Neurocognitive individualized training versus social skills individualized training: a randomized trial in patients with schizophrenia. Schizophr Res 2013;150:69-75.

18 Pedrero-Perez EJ, Rojo-Mota G, Ruiz-Sanchez de Leon JM, et al. Cognitive remediation in addictions treatment. Rev Neurol 2011;52:163-72.

19 Fals-Stewart W, Lucente S. The effect of cognitive rehabilitation on the neuropsychological status of patients in drug abuse treatment who display neurocognitive impairment. Rehabil Psychol 1994;39:75-94.

20 Bickel WK, Yi R, Landes RD, et al. Remember the future: working memory training decreases delay discounting among stimulant addicts. Biol Psychiatry 2011;69:260-5.

21 Rupp Cl, Kemmler G, Kurz M, et al. Cognitive remediation therapy during treatment for alcohol dependence. J Stud Alcohol Drugs 2012;73:625-34.

22 Peterson MA, Patterson B, Pillman BM, et al. Cognitive recovery following alcohol detoxification: a computerised remediation study. Neuropsychol Rehabil 2002;12:63-74.

23 Wiers RW, Eberl C, Rinck M, et al. Retraining automatic action tendencies changes alcoholic patients' approach bias for alcohol and improves treatment outcome. Psychol Sci 2011;22:490-7.

24 Volkow ND, Morales M. The brain on drugs: from reward to addiction. Cell 2015;162:712-25.

25 Tang Y-Y, Posner MI, Rothbart MK, et al. Circuitry of self-control and its role in reducing addiction. Trends Cogn Sci 2015;19:439-44.

26 Noël X, Van der Linden M, Brevers D, et al. Separating intentional inhibition of prepotent responses and resistance to proactive interference in alcohol-dependent individuals. Drug A/cohol Depend 2013;128:200-5.

27 Yücel M, Oldenhof E, Ahmed SH, et al. A transdiagnostic dimensional approach towards a neuropsychological assessment for addiction: an international Delphi consensus study. Addiction 2019;114:1095-109.

28 Penolazzi B, Del Missier F, Stramaccia DF, et al. Testing the transdiagnostic hypothesis of inhibitory control deficits in addictions: an experimental study on gambling disorder. J Behav Addict 2020;9:339-46.

29 Mathieu E, McGeechan K, Barratt A, et al. Internet-based randomized controlled trials: a systematic review. J Am Med Inform Assoc 2013;20:568-76.

30 Bowie CR, Gupta M, Holshausen K, et al. Cognitive remediation for treatment-resistant depression: effects on cognition and functioning and the role of online homework. J Nerv Ment Dis 2013;201:680-5.

31 Leeman RF, Potenza MN. Similarities and differences between pathological gambling and substance use disorders: a focus on impulsivity and compulsivity. Psychopharmacology 2012;219:469-90.

32 Ferris J, Wynne H. The Canadian problem gambling index: final report. Canadian Centre on Substance Abuse 2001.

33 Pickering D, Keen B, Entwistle G, et al. Measuring treatment outcomes in gambling disorders: a systematic review. Addiction 2018;113:411-26.

34 Whiteside SP, Lynam DR. The five factor model and impulsivity: using a structural model of personality to understand impulsivity. Pers Individ Dif 2001;30:669-89. 
35 Billieux J, Rochat L, Ceschi G, et al. Validation of a short French version of the UPPS-P impulsive behavior scale. Compr Psychiatry 2012;53:609-15.

36 EuroQol Group. EuroQol--a new facility for the measurement of health-related quality of life. Health Policy 1990;16:199-208.

37 Luquiens A, Whalley D, Crawford SR, et al. Development of the alcohol quality of life scale (AQoLS): a new patient-reported outcome measure to assess health-related quality of life in alcohol use disorder. Qual Life Res 2015;24:1471-81.

38 Eagle DM, Baunez C, Hutcheson DM, et al. Stop-signal reaction-time task performance: role of prefrontal cortex and subthalamic nucleus. Cereb Cortex 2008;18:178-88.

39 Sekhon M, Cartwright M, Francis JJ. Acceptability of healthcare interventions: an overview of reviews and development of a theoretical framework. BMC Health Serv Res 2017;17:88.

40 Simons M, Kursawe A-L. Metacognitive therapy for posttraumatic stress disorder in youth: a feasibility study. Front Psychol 2019;10:264.
41 Melville KM, Casey LM, Kavanagh DJ. Psychological treatment dropout among pathological gamblers. Clin Psychol Rev 2007;27:944-58.

42 Jimenez-Murcia S, Aymamí N, Gómez-Peña M, et al. Does exposure and response prevention improve the results of group cognitivebehavioural therapy for male slot machine pathological gamblers? $\mathrm{Br}$ J Clin Psychol 2012;51:54-71.

43 Goudriaan AE, Yücel M, van Holst RJ. Getting a grip on problem gambling: what can neuroscience tell us? Front Behav Neurosci 2014;8:141.

44 Merkouris SS, Thomas SA, Browning CJ, et al. Predictors of outcomes of psychological treatments for disordered gambling: a systematic review. Clin Psychol Rev 2016;48:7-31.

45 Szerman N, Peris L. Precision psychiatry and dual disorders. J Dual Diagn 2018;14:237-46. 